\title{
MOLECULAR GENOTYPING OF CHICKEN (Gallus gallus L.) FEATHERING GENES IN CONNECTION WITH SEPARATION BY SEX
}

\author{
Ya.I. ALEKSEEV ${ }^{1,}$, A.M. BORODIN ${ }^{3}$, A.V. NIKULIN', Zh.V. EMANUILOVA ${ }^{4}$, \\ D.N. EFIMOV ${ }^{4}$, V.I. FISININ ${ }^{5}$
}

\begin{abstract}
${ }^{1}$ All-Russian Research Institute of Agricultural Biotechnology, Federal Agency of Scientific Organizations, 42, ul. Timiryazevskaya, Moscow, 127550 Russia, e-mail jalex@iab.ac.ru (corresponding author);

${ }^{2} J S C$ «Syntol», 42, ul. Timiryazevskaya, Moscow, 127550 Russia, e-mail jalex@syntol.ru;

${ }^{3}$ Non-proffn Partnership Institute of Medico-Biological Research, 10, ul. Studenaya, Nizhnii Novgorod, 603000 Russia, e-mail Aborodinm@sinn.ru;

${ }^{4}$ Breeding and Genetic Center «Smena», Federal Agency of Scientific Organizations, pos. Bereznyaki, Moscow Province, 141327 Russia, e-mail Smena@tsinet.ru;

${ }_{5}^{5}$ Federal Scientific Center All-Russian Research and Technological Poultry Institute RAS, Federal Agency of Scientific Organizations, 10, ul. Ptitsegradskaya, Sergiev Posad, Moscow Province, 141315 Russia, e-mail olga@vnitip.ru, vnitip@vnitip.ru

ORCID: Fisinin V.I. orcid.org/0000-0003-0081-6336

The authors declare no conflict of interests

Acknowledgements:

The study was carried out with the equipment of «Biotechnology» center (All-Russian Research Institute of Agricultural Biotechnology).

Supported financially by Federal Agency of Scientific Organizations, the additional state assignment for Breeding and Genetic Center «Smena» № 007 of August 5, 2016 in the framework of the Federal Scientific and Technical Program of agricultural development for 2017-2025 (Sub 2 «Breeding competitive crosses of domestic meat poultry»)

Received October 5, 2016
\end{abstract}

\section{Abstract}

Traditional breeding is time and material consuming. Modern laboratory techniques significantly speed up and reduce the costs for breeding animals with the desired properties. A test based on the quantitative real-time PCR (qPCR) technology was developed to distinguish between homozygous and heterozygous state of the genes from alleles $K$ and $k$ which are responsible for the rate of wing feather growth in day-old chicks. The use of quantitative real-time PCR for the analysis of genotypes is aimed at the discrimination between one and two copies of the target gene in a genome. To obtain reliable results, certain rules must be followed when conducting the assay: the efficiency of the PCR should be close to the maximum; it is possible to obtain a significant number of false results without the appropriate statistical analysis. A new assay algorithm was proposed to overcome the limitations of qPCR: all samples are subjected to two successive independent analyses in parallel with the reference samples of both genotypes; if the two runs produce divergent results then the assay is repeated and the previous results are discarded. This approach allows to reduce assay error probability down to zero. The new system consists of three (instead of four) primers for amplification of two genes and two probes, allowing efficient analysis of various allele $K$ genotypes. Quantitative realtime PCR data analysis was performed by $\Delta \Delta \mathrm{Ct}$ method using the statistics software package SPSS for ROC analysis. Using the method developed, the percentage of $K K, K k$ and $k k$ genotypes was determined in 145 cocks of original lines B5, B6, B7 and B9 of domestic meat chicken of cross Smena 8. It was shown that 19 cocks of line B5 and 15 cocks of line B6 had $k k$ genotype. From the 46 cocks of line B7, none had $k k$ genotype, 17 cocks (37\%) had $K k$ genotype, and 29 cocks (63\%) had $K K$ genotype. From the 65 cocks of line B9, none had $k k$ genotype, 17 cocks $(26 \%)$ had $K k$ genotype, and 48 cocks $(74 \%)$ had $K K$ genotype. Analyzed fragments were sequenced to exclude the effects of possible nucleotide sequence variability on the assay. The sequences did not contain any nucleotide substitutions in the sites of the primers and probes annealing. The data obtained will accelerate selection of new domestic meat chicken breeds with possibility of sexing based on feather length in day-old chicken. Further breeding work involves the assessment of the offspring using traditional and molecular genetic methods.

Keywords: real-time polymerase chain reaction, qPCR, genotype, gene copy number, autosex chickens, poultry selection, meat chickens

Gender identification (sexing), using simple phenotypic techniques (for example, differentiation according to the growth rate of feathers of the wing, according to the color of the feathers), gives a number of advantages in compari- 
son to the traditional method, in which the presence of genital tubercles is determined. The solution of this urgent task will significantly reduce the requirements for personnel, the percentage of errors and allow rapid of sorting of chickens. For broiler sexing, a different rate of wing feathering is used in chickens of the opposite sex at 1-day age [1-3]. The genetics of this phenomenon is well studied [4-6]. The allele of slow feathering $K$ is located on the $\mathrm{Z}$ chromosome and is linked to the gender. Cocks in the genome have two male sex chromosomes ZZ, and hens have one male Z and one female W. Sex of chickens at 1-day age can be determine by a development of the wing feathers, which is inherited through sex-linked alleles, dominant $K$ and recessive $k$ (rapid feathering) [7]. In 1-day-old chickens having the genotype $K K, K k$ or $K w$, the formation of the feather cover of the wing is slow (the feathers are weakly developed, they are either the same in length or the covering feathers are longer than the flight feathers), in individuals with $k k$ or $k w$ genotypes, the flight feathers of the wing are well developed, and the covering feathers are shorter than the flight feathers. Crossing cocks homozygous for the fast feathering gene $k k$ and hens with the gene of slow feathering $K w$ leads to offspring, among which all chickens with fast wing feathering are females $k w$, and chickens with slow feathering are males $K k$.

A detailed study of the $K$ gene located in the integration region of the endogenous retrovirus ev21 made it possible to establish a relationship of the doubling of the tandem partially deleted genes of the flagellar sperm protein $d$ Spef 2 and the prolactin receptor $d P r l r$ with the $K$ genotype [8]. A study of the mechanisms determining slow feathering showed the importance of expression of the products of the $d S p e f 2$ and $d P r l r$ genes in the skin $[9,10)$. The prolactin receptor, whose ligand is the pituitary hormone prolactin, belongs to the family of receptors of cytokines and is expressed in all tissues of vertebrates. About 300 different functions are attributed to prolactin, including the maintenance of water-salt balance, participation in growth and development, metabolism, hormonal and brain activity, control of behavior, regulation and functioning of the immune system [11], reproduction, egg production in hens (12), induction of molting [13].

The peculiarity of the domestic cross Smena 8 is its universality. With a stable high rate of growth of young animals, which allows reducing the time of growing, the bird has a stable reproduction, is well adapted to Russian conditions (unlike foreign crosses) and both cage and floor housing technologies. The parent flock has a high egg-laying peak $(85 \%)$. The cross is also characterized by good viability, uniformity of the flock, high yield of pectoral and leg muscles, effective feed conversion (http://www.gossort.com/docs/rus/REESTR_SKOT2015.pdf).

The production of autosexing chickens with different feathering at 1-dayold age (by the example of the crossing of B7 cocks and chicks and B9 hens of the meat cross Smena 8) is illustrated by the scheme:

\begin{tabular}{|c|c|c|}
\hline Line & \multicolumn{2}{|c|}{$\mathrm{B} 7\left({ }^{\Uparrow}\right) \times \mathrm{B} 9($ () } \\
\hline Genotype & $k k$ & $K w$ \\
\hline Trait & Fast wing feathering & Slow wing feathering \\
\hline $\begin{array}{l}\text { Offspring } \\
\text { Genotype }\end{array}$ & $K k(\lambda)<$ & 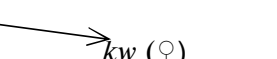 \\
\hline Trait & Slow wing feathering & $\begin{array}{c}\kappa w(+) \\
\text { Fast wing feathering }\end{array}$ \\
\hline
\end{tabular}

Sexing is necessary for separated broiler farming. This technology provides more efficient use of feed, better survival, increased similarity in weight, that is, improves the output of broilers at lower economic costs.

We have proposed a new test system that allows to determine the number of copies of the gene responsible for the rate of feathering in chicks, as well as the algorithm for analysis of gene copy number by quantitative real-time PCR. This makes it possible to genotype $K$ and $k$ chicks effectively and with high throughput 
to accelerated production of the original meat cross lines of the Smena 8 giving autosexing offspring.

The aim of the study was to develop a test for the molecular typing of fast and slow feathering genes in chickens using real-time PCR (PCR-RT).

Technique. For development of the test system and the genotyping algorithm we used 145 DNA samples of cocks of B5, B6, B7 and B9 domestic meat lines of cross Smena 8.

DNA was isolated from the selected feathers using the M-Sorb set (ZAO Sintol, Russia). For sample preparation, $0.5 \mathrm{~cm}$ tip of the quill was placed in a $1.5 \mathrm{ml}$ test tube (OOO Tekhosnastka, Russia). A $400 \mu$ lysis solution was added and incubated at $37^{\circ} \mathrm{C}$ for $20 \mathrm{~min}$ with stirring, the lysate was precipitated in a high-speed microcentrifuge Cyclotemp-902 (ZAO Cyclotemp, Russia) for $3 \mathrm{~min}$ at $13,000 \mathrm{rpm}$. The supernatant was transferred to a $1.5 \mathrm{ml}$ test tube and isolation continued according to the standard M-Sorb set protocol. The DNA concentration was determined on a NanoPhotometer spectrophotometer (Implene, Germany). About $3 \mu \mathrm{g}$ of DNA was taken into the reaction $\left(\mathrm{A}_{260} / 280=1.8-2.0\right)$. The primers and probes for PCR-RT and sequencing presented below are based on known DNA sequences [9, 10, 14] and synthesized in ZAO Synthol; fluorescent labels were carboxyfluorescein (FAM) and 6-carboxyrodamine (R6G) with the $3^{\prime}$-terminal modification of phosphate $(\mathrm{p})$ :

$\begin{array}{ll}\text { LFBF1 } & 5^{\prime} \rightarrow 3^{\prime}: \text { CTCACTGAAACCATCCCTGGA } \\ \text { LFBR1 } & 5^{\prime} \rightarrow 3^{\prime}: \text { CTAACCTGAGACAAGTGTCGGA } \\ \text { LFBV1 (зонд) } & 5^{\prime} \rightarrow 3^{\prime}:(6 F A M)-C C C C T T A A A T G C C(d T-B H Q 1) T G C T T T T C C A T C-p \\ \text { NFR } & 5^{\prime} \rightarrow 3^{\prime}: \text { CTGTGGTTTGCTCGGTTTTGGA } \\ \text { NFP (зонд) } & 5^{\prime} \rightarrow 3^{\prime}: \text { (5R6G)-CTCCATCTCTCC(dT-BHQ1)TGCTTTTCCATC-p } \\ \text { PROSF } & 5^{\prime} \rightarrow 3^{\prime}: \text { GTTTGCCTATCACAGCATTGTAGA } \\ \text { PROSR } & 5^{\prime} \rightarrow 3^{\prime}: \text { GCTGGTTCCTCATCCTGTCTGA } \\ \text { SPEFR } & 5^{\prime} \rightarrow 3^{\prime}: \text { GTGCAATTTAGCAGTGCATGTGA }\end{array}$

Multiplex PCR-RT (with FAM and R6G detection channels) was performed on an ANK-32M (Institute of Analytical Instrument Engineering, Russia) using the following temperature-time cyclogram (40 cycles): denaturation at $93{ }^{\circ} \mathrm{C}$ for $15 \mathrm{sec}$, annealing at $60{ }^{\circ} \mathrm{C}$ for $30 \mathrm{sec}$. A reaction mixture $(20 \mu \mathrm{l})$ was used for PCR-RT (ZAO Sintol, Russia). The primer concentration in the reaction mixture was $450 \mathrm{nM}$, the probe concentration was $250 \mathrm{nM}$. The specificity of PCR-RT was confirmed by sequencing the amplification products obtained with primers PROSF, PROSR and SPEFR, on a Nanofor 05 genetic analyzer (Institute of Analytical Instrument Engineering, Russia) in accordance with the manufacturer's protocol.

Results. The primer system LFBF1, LFBR1 and the LFBV1 probe allowed the detection of the $d$ Spef $2 d P r l r$ gene, the primer system LFBF1, NFR, and the NFP probe revealed the reference Prlr chicken prolactin receptor gene. Primers PROSF, PROSR and SPEFR were used for sequencing fragments of the genes Spef2 and Prlr.

The determination of genotypes $K K$ and $K k$ by PCR-RT is reduced to the problem of discrimination of two copies of a gene in the genome from one $[8,15,16]$. It can be solved by quantitative PCR $[8,17]$, as well as by hybridization of nucleic acids $[15,18,19]$. Each of these approaches has advantages and disadvantages. Southern hybridization is laborious, takes a lot of time and requires a variety of equipment. When quantitative PCR (PCR-RT) is used to solve the task, the results may be inadequate if the necessary conditions are not met during the test and subsequent statistical processing of the data $[15,18,19]$. However, with appropriate execution, this method is highly technological and allows for a short time to accumulate a large array of information. For reliable testing it is necessary that in one cycle of PCR the amount of DNA is doubled and 
the amplification efficiency approaches the maximum [20, 21]. Also, you need to carefully monitor its probable fluctuations, which can lead to incorrect results.

The selected primers and probes were analyzed in multiplex PCR-RT for two samples (No. 64 and No. 65) in a series of five 2-fold DNA dilutions in two replicates. The amplification efficiency of the dSpef2 dPrlr gene was $92 \%$ $\left(\mathrm{R}^{2}=0.9945\right)$ with $94 \%$ for the reference prolactin gene $\left(\mathrm{R}^{2}=0.9991\right)$. The use of one common LFBF1 primer to amplify two genes makes the proposed system more robust by reducing the number of components and smooths possible differences in primer performance.

In preliminary experiments to determine the genotypes of chicks, the samples were examined in 2-fold replicates. Up to $10 \%$ of the samples in one of the experiments yielded results not only lying in the so-called "gray" area, but also reliably opposite in the repetition of one sample. To increase the reliability of the test, we developed a new algorithm that differs from that described earlier [8]. For each batch of examined samples, it is mandatory to use at least four reference samples for each of those genotypes that are being analyzed. Then a reindependent determination is made with a similar set of samples. The results in each of the two tests are counted, and when any deviation is detected, a new definition with reference samples in two independent repeats is performed. Such an algorithm makes it possible to reduce the error probability to a minimum.

Using a new algorithm, 19 B5 samples of the DNA of the cocks from the line and 15 B6 were analyzed. In $100 \%$ of cases, the genotype $k k$ was confirmed, which completely correlated with the phenotype for these two lines.

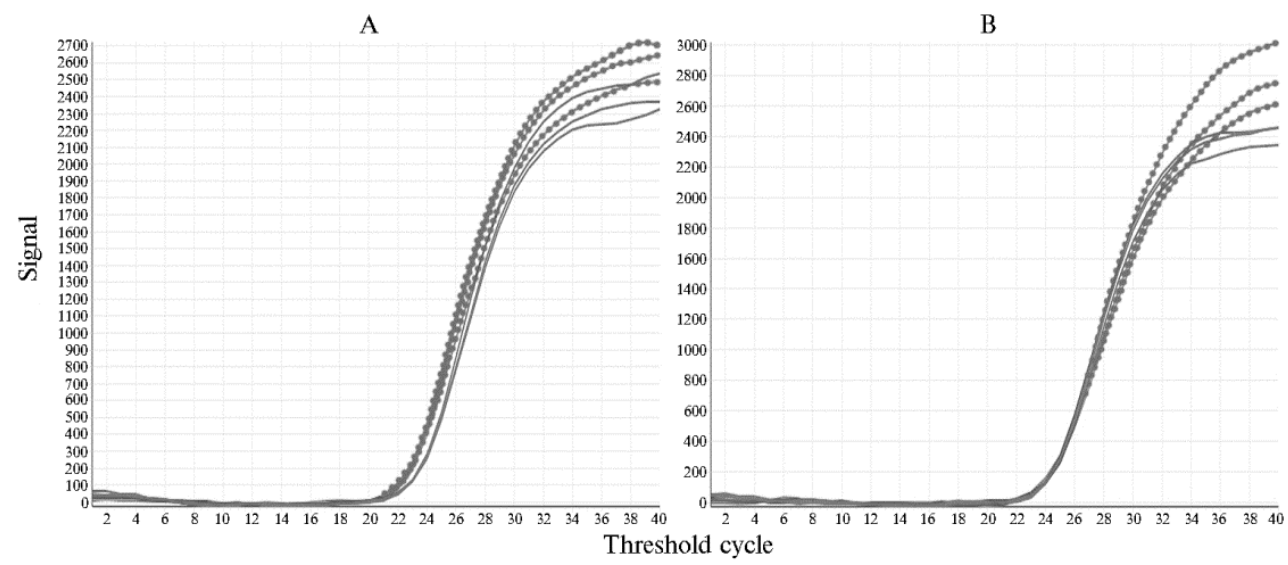

The result of genotyping cocks of the $\mathbf{B} 7$ cross-line Smena 8 using real-time PCR: A - sample No. $64(\Delta \mathrm{Ct}=0.8)$ for genotype $K K, \mathrm{~B}-$ sample No. $65(\Delta \mathrm{Ct}=0.02)$ for genotype $K k$; «-»detection channel FAM (dSpef2 dPrlr), «— - - detection channel R6G (Prlr).

To study the cocks of the lines B7 and B9, four reference cocks with the $K K$ genotype and four with the genotype $K k$ were used. Examples of the kinetic curves of PCR amplification obtained for samples No. 64 and No. 65 of B7 are shown in the figure. For each analyzed sample, the difference in the threshold reaction cycles for the tandem $d$ Spef $2 d$ Prlr (detection channel FAM) and the prolactin gene (detection channel R6G) was calculated by the formula $\Delta \mathrm{Ct}=\mathrm{Ct}$ (FAM) - Ct (R6G). As can be seen from the figure, the threshold cycles of amplification of the fragment of the reference gene of the prolactin chicks (detection channel R6G) are greater than the threshold amplification cycles of the fragment of the dSpef2 dPrlr gene (the detection channel FAM) (see Fig., A), or equal to them (see Fig., B). The difference is explained by the fact that DNA samples of individuals having the $K K$ genotype contain twice as many copies of the $K$ gene as in the case of cocks with the genotype $K k$. The sample genotypes 
were analyzed using the $\Delta \Delta \mathrm{Ct}$ [22] method in accordance with the description [8], the data was processed with the SPSS statistics package [23] using the ROCanalysis [24]. The use of other binary classifiers [25] and statistical methods [18] to increase the reliability of results can be promising in such studies.

The results of the study of samples from 46 cocks of the line B7 showed that none of the genotypes were identified as $k k, 17$ (37\%) had the genotype $K k$ and $29(63 \%)$ had the genotype $K K$. According to the analysis of samples from 65 cocks of the line B9, none has the genotype $k k$, in 17 (26\%) the genotype $K k$ was found and in $48(74 \%)$ the genotype $K K$ was found. To exclude the possible influence of the variability of the investigated gene sections, sequencing of the DNA amplification products obtained with primer pairs PROSF and PROSR (Prlr gene), as well as PROSF and SPEFR (dSpef2 dPrlr gene) was carried out. It was found that the sequences in the analyzed regions are generally invariant and do not contain nucleotide substitutions in the annealing areas of primers and probes.

In the future it is supposed to estimate the population by traditional and molecular genetic methods. According to the results, the cocks of the B7 line with the genotypes $K K$ and $K k$, as well as the cocks of the B9 line with the genotypes $K k$ and $k k$ and their descendants will be excluded from the selection, which will allow obtaining lines with a given genotype.

Thus, a test was developed and an algorithm was proposed for detecting in 1-day chickens the homo- and heterozygous state of sex-linked $K$ and $k$ alleles associated with the wing feather growth rate, using quantitative real-time PCR. With the help of the proposed test, the percentage of genotypes $K K, K k$ and $k k$ was determined among 145 cocks of the lines B5, B6, B7 and B9 of the domestic meat cross Smena 8. Further selection work involves the evaluation of the bird by traditional and molecular genetic methods to exclude from the selection the cocks of line B7 with genotypes $K K$ and $K k$, cocks of line B9 with genotypes $K k$ and $k k$, and also their descendants as not corresponding to the target parameters.

\section{R E F E R E N C ES}

1. S e re brovsky A.S. Crossing-over involving three sex-linked genes in chickens. Amer. Nat., 1922, 56: 571-572 (doi: 10.1086/279898).

2. Wa r re n D.C. Inheritance of rate of feathering in poultry. J. Hered., 1925, 16(1): 13-18 (doi: 10.1093/oxfordjournals.jhered.a102502).

3. Som es R.G. Jr. Delayed feathering, a third allele at the $K$ locus in the domestic fowl. $J$. Hered., 1969, 60(5): 281-288 (doi: 10.1093/oxfordjournals.jhered.a107994).

4. Jones D.G., Hutt F.B. Multiple alleles affecting feathering in the fowl. J. Hered., 1946, 37(7): 197-205 (doi: 10.1093/oxfordjournals.jhered.a105617).

5. Warren D.C. Retarded feathering in the fowl. A new factor affecting manner of feathering. J. Hered., 1933, 24(11): 431-434 (doi: 10.1093/oxfordjournals.jhered.a103691).

6. W a r re n D.C. Developing early-feathering strains in heavy breeds of poultry. Agricultural Experiment Station, Kansas State College of Agriculture and Applied Science, 1944.

7. M c Gibbo n W.H. A sex-linked mutation affecting rate of feathering in chickens. Poult. Sci., 1977, 56(3): 872-875 (doi: 10.3382/ps.0560872).

8. Elferink M.G., Valle e A.A.A., Jungerius A.P., Crooijmans R.P.M.A., Gro e $n$ e $n$ M.A.M. Partial duplication of the PRLR and SPEF2 genes at the late feathering locus in chicken. BMC Genomics, 2008, 9: 391 (doi: 10.1186/1471-2164-9-391).

9. Zha o J., Yao J., Li F., Yang Z., Sun Z., Qu L., Wang K., Su Y., Zhang A., Mo nt go me ry S.A., G e ng T., $\mathrm{Cu}$ i H. Identification of candidate genes for chicken early- and late-feathering. Poult. Sci., 2016, 95(7): 1498-1503 (doi: 10.3382/ps/pew131).

10. Bu G., Huang G., Fu H., Li J., Huang S., Wang Y. Characterization of the novel duplicated PRLR gene at the late-feathering $K$ locus in Lohmann chickens. J. Mol. Endocrinol., 2013, 51: 261-276 (doi: 10.1530/jme-13-0068).

11. B ole-F e y s o t C., G offi n V., Ed e ry M., B in art N., Kelly P.A. Prolactin (PRL) and its receptor: actions, signal transduction pathways and phenotypes observed in PRL recep- 
tor knockout mice. Endocrine Reviews, 1998, 19(3): 225-268 (doi: 10.1210/edrv.19.3.0334).

12. Cu i J.-X., D u H-L., Li ang Y., D e ng X.-M., Li N., Z hang X.-Q. Association of polymorphisms in the promoter region of chicken prolactin with egg production. Poult. Sci., 2006, 85(1): 26-31 (doi: 10.1093/ps/85.1.26).

13. Juhn M., Harris P.C. Molt of capon feathering with prolactin. Exp. Biol. Med., 1958, 98(3): 669-672 (doi: 10.3181/00379727-98-24146).

14. International Chicken Genome Sequencing Consortium. Sequence and comparative analysis of the chicken genome provide unique perspectives on vertebrate evolution. Nature, 2004, 432: 695-717 (doi: 10.1038/nature03154).

15. B u b n e r B., B a ld w in I.T. Use of real-time PCR for determining copy number and zygosity in transgenic plants. Plant. Cell Rep., 2004, 23: 263-271 (doi: 10.1007/s00299-004-0859-y).

16. M i e o g J.C., H ow it t C.A., R a l J.P. Fast-tracking development of homozygous transgenic cereal lines using a simple and highly flexible real-time PCR assay. BMC Plant Biol.. 2013, 13: 71 (doi: 10.1186/1471-2229-13-71).

17. Stefano B., Patrizia B., Mat te o C., Massimo G. Inverse PCR and quantitative pcr as alternative methods to Southern blotting analysis to assess transgene copy number and characterize the integration site in transgenic woody plants. Biochem. Genet., 2016, 54(3): 291305 (doi: 10.1007/s10528-016-9719-z).

18. Yuan J.S., Burris J., S t e wart N.R., M e nt e wab A., S tewart C.N. Jr. Statistical tools for transgene copy number estimation based on real-time PCR. BMC Bioinformatics, 2007, 8(Suppl 7): S6 (doi: 10.1186/1471-2105-8-S7-S6).

19. Huang Y., Y in X., Zhu C., Wang W., Grierson D., Xu C., Chen K. Standard addition quantitative real-time PCR (SAQPCR): a novel approach for determination of transgene copy number avoiding PCR efficiency estimation. PLOS ONE, 2013, 8(1): e53489 (doi: 10.1371/journal.pone.0053489).

20. Sochivko D.G., Fedorov A.A., Varla mov D.A., Ku rochkin V.E., Pet rov R.V. Simulation of the PCR amplification as two-type-particle branching process. Dokl. Biochem. Biophys., 2010, 434: 239-241 (doi: 10.1134/S1607672910050054).

21. Sochivko D.G., Fedorov A.A., Lavrov V.V., Varlamov D.A., Kurochkin V.E., Petrov R.V. Stochastic modeling of polymerase chain reaction kinetic curves. Dokl. Biochem. Biophys., 2011, 439: 188-191 (doi: 10.1134/S1607672911040119).

22. Livak K.J., Schmittgen T.D. Analysis of relative gene expression data using real-time quantitative PCR and the $2^{-\Delta \Delta} C_{\mathrm{T}}$ method. Methods, 2001, 25(4): 402-408 (doi: 10.1006/meth.2001.1262).

23. Bryman A., Crame r D. Quantitative data analysis with IBM SPSS 17, 18 and 19: A guide for social scientists. Routledge, NY, 2011 (ISBN 978-0-415-57918-6).

24. Fawcett T. An introduction to ROC analysis. Pattern Recognition Letters, 2006, 27(8): 861874 (doi: 10.1016/j.patrec.2005.10.010).

25. Saito T., Rehmsmeier M. The precision-recall plot is more informative than the ROC plot when evaluating binary classifiers on imbalanced datasets. PLoS ONE, 2015, 10(3): e0118432 (doi: 10.1371/journal.pone.0118432). 